\title{
Alcohol energy intake and habitual physical activity in older adults
}

\author{
Klaas R. Westerterp ${ }^{1 *}$, Erwin P. Meijer ${ }^{1}$, Annelies H. C. Goris ${ }^{1}$ and Arnold D. M. Kester ${ }^{2}$ \\ ${ }^{1}$ Department of Human Biology and \\ ${ }^{2}$ Department of Methodology and Statistics, Maastricht University, PO Box 616, 6200 MD Maastricht, \\ The Netherlands
}

(Received 5 March 2003 - Revised 30 July 2003 - Accepted 2 September 2003)

\begin{abstract}
Alcohol forms a significant component of many diets and it supplements rather than displaces daily energy intake. Surprisingly, alcohol intake does not systematically increase body weight. The present study assessed whether a higher level of habitual physical activity in the daily environment is associated with a higher alcohol intake. Alcohol intake as part of total food intake was measured with a $7 \mathrm{~d}$ dietary record while at the same time physical activity was monitored with a tri-axial accelerometer for movement registration. Subjects were twenty women and twenty-four men, aged $61 \pm 5$ years, of BMI $27 \cdot 1 \pm 4.6 \mathrm{~kg} / \mathrm{m}^{2}$. Between subjects, there was a positive association between the level of habitual physical activity and alcohol intake $(r 0.41 ; P<0.01)$. The subjects with higher alcohol intake had a higher activity level. On days with and days without alcohol consumption there was no difference in physical activity within subjects. In conclusion, it was shown that subjects with higher alcohol consumption are habitually more active. This may explain the lack of increasing body weight through additional energy intake from alcohol.
\end{abstract}

Energy balance: Overweight: Accelerometers: Alcohol paradox

Nationwide epidemiological studies show a paradoxical association between alcohol intake and body weight. While alcohol energy is additive to the normal diet there is no positive correlation with body weight (Colditz et al. 1991). Alcohol is a significant component of the diet in many countries. Per capita alcohol consumption is about $3-9 \%$ of daily energy intake (Kant, 2000). Alcohol seems to supplement rather than displace food-derived energy (Rose et al. 1995). Studies on the effects of alcohol consumption on food consumption have led to the conclusion that alcohol consumption is associated with minimal compensatory down regulation of energy intake from other foods (Foltin et al. 1993; Tremblay et al. 1995b; Poppitt \& Prentice, 1996). Even increased energy intake after an aperitif, due to decreased control, caused by light-headedness, is not compensated for (Westerterp-Plantenga \& Verwegen, 1999). From studies on alcohol intake and body weight, a bias could be postulated. Subjects who are overweight systematically under-report energy intake more than lean subjects (Westerterp \& Goris, 2002). Thus, the absence of the expected positive relationship between alcohol intake and body weight could be due to under-reporting in overweight subjects.

The lack of a relationship between the apparent alcohol energy surplus and BMI in epidemiological studies has led to the suggestion that alcohol energy has a low metabolisable energy defined as gross energy minus energy loss in faeces and urine (Colditz et al. 1991). However, ethanol is readily absorbed and is principally eliminated by metabolism in the liver, urinary losses being about $0.3 \%$ of the dose (Holford, 1987). Since ethanol is not stored in the body, it must be oxidised preferentially to other fuels. Thus, alcohol ingestion results in a shortterm reduction of fat oxidation (Suter et al. 1992). Controlled feeding studies have shown that ethanol has the same metabolisable energy as carbohydrate (Rumpler et al. 1996).

In controlled studies as mentioned earlier, the lifestyle of the subjects has not been taken into account. In the present study, it is suggested that the level of physical activity is associated with alcohol intake. The present study therefore assessed habitual physical activity and habitual alcohol intake in the daily environment, hypothesising that the addition of alcohol to energy intake might be offset by a higher physical activity-induced energy expenditure.

\section{Methods \\ Healthy adults (twenty women and twenty-four men, aged $61 \pm 5$ years, of BMI $27 \cdot 1 \pm 4.6 \mathrm{~kg} / \mathrm{m}^{2}$ ) participated in the study. The subjects were recruited from advertisements in the local media. Status varied from lower to upper}


socio-economic class. Detailed information concerning the study was provided and informed consent was obtained. The medical ethics committee of Maastricht University approved the study.

Food intake was measured with a $7 \mathrm{~d}$ dietary record (Goris et al. 2001). Subjects received instructions from a dietitian on how to keep a food record and were asked not to change their habitual food intakes. Amounts consumed were recorded in household units, by volume or by weight. Recipes of meals were also recorded. The data were processed with a computer program based on food tables (BECEL NUTRITION PROGRAM, 1988; Nederlandse Unilever bedrijven BV, Rotterdam, The Netherlands).

Physical activity was measured over the same week with a tri-axial accelerometer for movement registration (Tracmor; Maastricht University, Maastricht, The Netherlands) (Meijer et al. 2001). The Tracmor is a portable motion sensor consisting of a body-fixed tri-axial accelerometer and a data unit for on-line registration, processing and storage of acceleration signals. The Tracmor $(70 \times 28 \times 8 \mathrm{~mm}, 30 \mathrm{~g})$ is composed of three uni-axial piezoelectric accelerometers and registers accelerations in three orthogonal directions during body movement. Acceleration signals are processed to obtain the sum of the rectified and integrated acceleration curves from all three measurement directions. The time for integration is set at $1 \mathrm{~min}$ and the finally obtained output from the Tracmor is expressed as kCounts/d (Bouten et al. 1996). Subjects were instructed to wear the Tracmor during waking hours, except during bathing and showering. Tracmor output was expressed as kCounts and converted to energy expenditure with an equation based on simultaneous measurement of total energy expenditure with doubly labelled water in a subgroup of the study population (Goris et al. 2001). Resting energy expenditure was measured with a ventilated hood system after an overnight fast in the morning at 06.45 hours. After a period of $15 \mathrm{~min}$ under thermoneutral temperature conditions in a supine position, resting metabolic rate was measured for at least $15 \mathrm{~min} . \mathrm{O}_{2}$ consumption and $\mathrm{CO}_{2}$ production were measured by means of a computerised, open-circuit, ventilated hood system. Gas analyses were performed using a paramagnetic $\mathrm{O}_{2}$ analyser (Servomex Type 500A; Crowborough, Sussex, UK) and an infrared $\mathrm{CO}_{2}$ analyser (Servomex Type 12-XI). The system was similar to the analysis system for the respiration chamber as described previously (Schoffelen et al. 1997). The calculation of resting metabolic rate was based upon the Weir formula (Weir, 1949).

The relationship of alcohol intake and physical activity level was analysed using linear regression. The activity level as a function of alcohol intake was analysed with correction for the potential confounders of age, sex and BMI. The validity of the obtained model was assessed using residual plots and a q-q plot of the residuals. If necessary the activity levels were transformed using a logarithm. Individual data points were checked for undue influence using Cook's distance. The corrected association of activity and alcohol intake was expressed as a partial correlation coefficient. Statistical significance was accepted at $P<0.05$.

\section{Results}

The mean energy intake was 8.8 (SD 2.3) $\mathrm{MJ} / \mathrm{d}$ and consisted of 16 (SD 3) \% energy from protein, 36 (SD 5) \% energy from fat, 43 (SD 6) \% energy from carbohydrate, and 6 (SD 4) \% energy from alcohol. Alcohol intake was negatively related to carbohydrate intake $(r-0.68$; $P<0.0001)$ and not related to fat intake. Mean alcohol intake ranged from 0 to $55 \mathrm{~g} / \mathrm{d}$. There was no association between alcohol intake and BMI $(r-0.18 ; P=0.24)$. If any, there was a tendency for a lower BMI at higher alcohol intake instead of the opposite. The physical activity level (PAL) of the subjects (total energy expenditure as a multiple of resting energy expenditure) ranged from 1.53 to 1.83. Physical activity and alcohol intake were not different for women and men, respectively, 21 (SD 6) $v$. 22 (SD 9) kCounts/d and 5 (SD 4) v. 6 (SD 5) \% of total energy intake. Between subjects, there was a positive association between alcohol intake and the level of habitual physical activity. Subjects with a higher activity level had a higher alcohol intake (Fig. 1). The unadjusted correlation of activity and alcohol intake was $0.40(P=0 \cdot 006)$. When corrected for age, sex and BMI, the partial correlation was $0.37(P=0 \cdot 017)$. The validity of this model appeared to be questionable, however, because of a non-linear $\mathrm{q}-\mathrm{q}$ plot of the residuals and because of an apparently non-constant variance of the residuals. Therefore, the activity variable counts/d was log-transformed and the analysis was repeated. Now, there were no more obvious model violations. The Cook's distance gave, with a maximal value of $0 \cdot 15$, no indication of overly influential points. The partial correlation, corrected for age, sex and BMI, was 0.38 $(P=0 \cdot 013)$. The regression analysis implied that, on average, for a $3 \%$ increase in activity level there was one additional energy percent alcohol intake.

Thirty of the forty-four subjects showed days with and days without alcohol consumption in the observation period; three subjects did not drink at all and eleven subjects drank on all days. On days with and days without

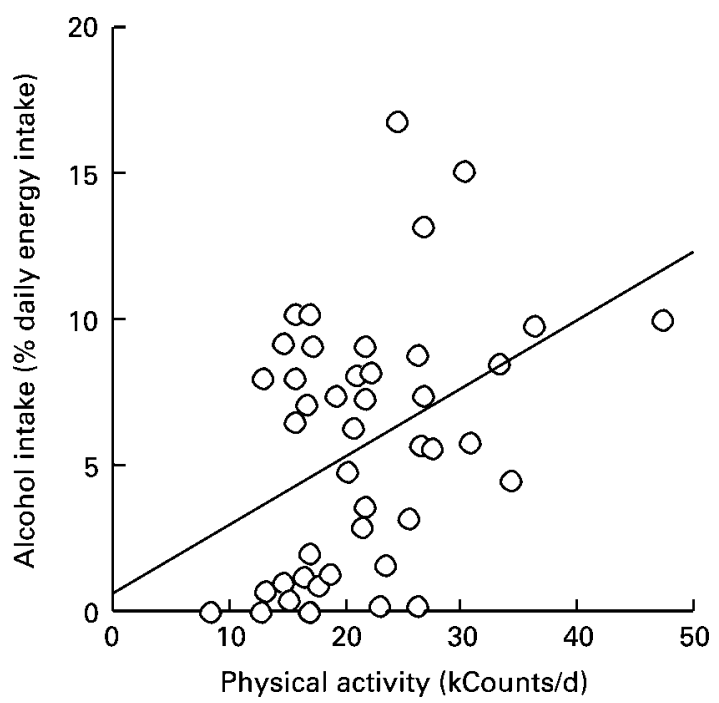

Fig. 1. Alcohol intake as a function of level of physical activity in forty-four healthy subjects, aged $61 \pm 5$ years and of BMI $27.1 \pm 4.6 \mathrm{~kg} / \mathrm{m}^{2}$, with the linear regression line $(r 0.41 ; P<0.01)$. 
alcohol consumption there was no difference in physical activity within subjects (Fig. 2).

\section{Discussion}

The mean alcohol intake was with a value of $5-6 \%$ of daily energy intake, equivalent to one to two standard servings of beer, wine or spirits per day, and representative of the per capita consumption in many countries. It is the level of moderate drinking, which has been associated with a lower total mortality, mainly by less CHD (Poikolanen, 1995; Rimm et al. 1996; Thun et al. 1997). The observed maximum alcohol intake of $55 \mathrm{~g} / \mathrm{d}$ or about five standard servings per day was close to the level of excessive drinking (Fleming et al. 1997). Of course, the interpretation of observations on alcohol consumption in daily life is complicated by many factors such as reporting errors and the confounding influence of other subject characteristics.

Studies with doubly labelled water have shown that under-reporting often biases measures of energy intake. Alcohol intake is even more liable to under-reporting. Nationwide surveys on alcohol consumption show that only $40-60 \%$ of the sales are covered, indicating that the error in self-report data on alcohol use can be substantial (Lemmens et al. 1992). In a subgroup of thirty subjects of the present study, the simultaneous measurement of total energy expenditure indicated an under-reporting of habitual energy intake of 16 (SD 16) \% (Goris et al. 2001). However, under-reporting seems to be of a linear nature. The ranking of individuals according to their self-reports is relatively stable across methods (Lemmens et al. 1992); only the real intake level will be systematically higher. Consequently, in relationships between alcohol consumption and, for example, health parameters, the real intake could be higher.

When alcohol is added to the normal diet it mainly reduces fat oxidation (Suter et al. 1992). Thus, it is surprising epidemiological studies show that alcohol intake does not systematically increase body weight and a higher

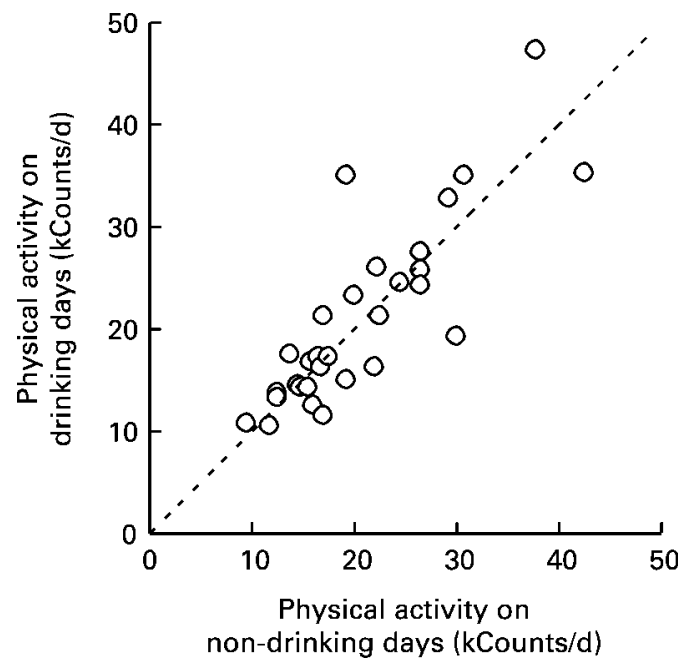

Fig. 2. Level of physical activity on days with alcohol consumption as a function of physical activity on days without alcohol consumption in thirty subjects, with the line of identity. physical activity is one of the few explanations. Indeed, subjects with a high alcohol intake and a low activity level show increased body fatness (Tremblay et al. 1995a).

Currently, the doubly labelled water method is generally accepted as the 'gold standard' for physical-activity assessment in free-living subjects. The method, however, cannot be applied at the population level, because ${ }^{18} \mathrm{O}$ is expensive and not readily available (Schoeller, 1999). Furthermore, the method can only be used to quantify the average level of physical activity over intervals of 1-3 weeks and does not provide information about the intensity, frequency, or duration of the physical activity. Consequently, the present study used tri-axial accelerometers as a method to assess the level of physical activity in free-living subjects. Accelerometers allow assessment of physical activity in larger populations over intervals that are long enough to be representative for normal daily life with minimal discomfort for the subjects (Bouten et al. 1996). Comparison between physical activity-generated accelerometer output and activity-associated energy expenditure as measured with the doubly labelled water method in thirty freeliving subjects over $7 \mathrm{~d}$ intervals revealed a significant relationship ( $r$ 0.80; Westerterp \& Bouten, 1997). Additionally, a subgroup of the subjects in the present study combined the accelerometer with the doubly labelled water method (Meijer et al. 2001). A correlation between accelerometer output and doubly labelled water-assessed PAL was shown $(r$ 0.78). Thus, tri-axial accelerometers are an objective and reliable tool that can be used to distinguish in activity levels between subjects.

The PAL of the subjects was in the lower part of the normal range of 1.2 to $2 \cdot 2-2 \cdot 5$, possibly because of their higher age of $61 \pm 5$ years. There is a steady decline of average activity level with increasing age (Westerterp \& Meijer, 2001). Starling et al. (1998), for example, reported a mean PAL of 1.68 (SD 0.28) in ninety-nine older men and women. Thus, the observed PAL of 1.53 to 1.83 reflects the actual PAL of an elderly population (Meijer et al. 2001).

The data do not indicate that alcohol drinking enhances physical activity. The similar activity scores during drinking and non-drinking days rather contraindicate this notion. Within subjects, alcohol intake had no effect on physical activity or vice versa. Furthermore, Rumpler et al. (1996) observed a similar effect on energy balance when alcohol compared with carbohydrate was administered, i.e. the same amount of metabolisable energy was supplemented with alcohol or carbohydrate.

The inter-individual association between alcohol intake and physical activity can offset the additive effect of alcohol consumption on energy intake. The energy value of the observed mean difference of alcohol intake between a subject with an activity score of $15 \mathrm{kCounts} / \mathrm{d}$ and $35 \mathrm{kCounts} /$ $\mathrm{d}$ is about one-third of the predicted difference in total daily energy expenditure (Goris et al. 2001). It confirms the earlier suggestion of Prentice et al. (1996) that a large proportion of the paradoxical inverse association between alcohol intake and BMI could be explained by such confounding variables such as age, smoking, social class, education and physical activity. The present study showed a key function for physical activity as an explanatory variable for the mentioned alcohol paradox. 
The explanation for the lack of a positive correlation between alcohol intake and body weight at the population level is that physically active subjects generally have higher alcohol consumption than more sedentary individuals. This has important consequences for the interpretation of the U-shaped relationship between alcohol and CHD (Klatsky, 1996).

The present study has shown that there is a relationship between activity level and alcohol intake, in a group of forty-four elderly subjects. This relationship remained when correcting for the potential confounders of age, sex and BMI. From a different point of view, it would be more plausible to model alcohol intake as a function of activity level. The resulting relationship when corrected for the same covariates as above would be just as significant, however. In fact, both analyses give the partial correlation of alcohol intake and activity level, partialised on (corrected for) age, sex and BMI. The causality of a relationship cannot be demonstrated in an observational study, and moreover, unmeasured confounders could in principle be the origin of the relationship.

In conclusion, the present study showed evidence for the lack of increasing body weight through additional energy intake from alcohol in that subjects having higher alcohol consumption are habitually more active.

\section{References}

Bouten CV, Verboeket-van de Venne WP, Westerterp KR, Verduin M \& Janssen JD (1996) Physical activity assessment: comparison between movement registration and doubly labeled water. J Appl Physiol 81, 1019-1026.

Colditz GA, Giovannucci E, Rimm EB, et al. (1991) Alcohol intake in relation to diet and obesity in women and men. $A m$ $J$ Clin Nutr 54, 49-55.

Fleming MF, Barry KL, Manwell LB, Johnson K \& London R (1997) Brief physician advice for problem alcohol drinkers: a randomized controlled trial in community-based primary care practices. JAMA 277, 1039-1045.

Foltin RW, Kelly TH \& Fischman MW (1993) Ethanol as an energy source in humans: comparison with dextrose-containing beverages. Appetite 20, 95-110.

Goris AHC, Meijer EP, Kester A \& Westerterp KR (2001) The use of a tri-axial accelerometer for the validity of reported food intake. Am J Clin Nutr 73, 549-553.

Holford NH (1987) Clinical pharmacokinetics of ethanol. Clin Pharmacokinet 13, 273-292.

Kant AK (2000) Consumption of energy-dense, nutrient-poor foods by adult Americans: nutritional and health implications. The third National Health and Nutrition Examination Survey, 1988-1994. Am J Clin Nutr 72, 929-936.

Klatsky AL (1996) Alcohol, coronary disease, and hypertension. Annu Rev Med 47, 149-160.

Lemmens PHHM, Tan ES \& Knibbe RA (1992) Measuring quantity and frequency of alcohol consumption. A comparison of 5 indices. J Stud Alcohol 53, 476-486.

Meijer EP, Goris AHC, Wouters L \& Westerterp KR (2001) Physical inactivity as a determinant of the physical activity level in the elderly. Int J Obes 25, 935-939.

Poikolanen K (1995) Alcohol and mortality: a review. J Clin Epidemiol 48, 455-465.

Poppitt AD \& Prentice AM (1996) Energy density and its role in the control of food intake: evidence from metabolic and community studies. Appetite 26, 153-174.

Prentice AM, Jebb SA \& Cole TJ (1996) Paradoxical associations between alcohol consumption and obesity in men and women. Int J Obes 20, Suppl. 4, S138.

Rimm EB, Klatsky A, Grobbee D \& Stampfer MJ (1996) Review of moderate alcohol consumption and reduced risk of coronary heart disease: is the effect due to beer, wine, or spirits? $\mathrm{Br}$ Med $J$ 312, 731-736.

Rose D, Murphy SP, Hudes M \& Viteri FE (1995) Food energy remains constant with increasing alcohol intake. J Am Diet Assoc 95, 698-700.

Rumpler WV, Rhodes DG, Baer DJ, Conway JM \& Seale JL (1996) Energy value of moderate alcohol consumption by humans. Am J Clin Nutr 64, 108-114.

Schoeller DA (1999) The shortage of O-18 water. Obes Res 7, 519.

Schoffelen PFM, Westerterp KR, Saris WHM \& Ten Hoor F (1997) A dual-respiration chamber system with automated calibration. J Appl Physiol 83, 2064-2072.

Starling RD, Toth MJ, Carpenter WH, Matthews DE \& Poehlman ET (1998) Energy requirements and physical activity in free-living older women and men: a doubly labeled water study. J Appl Physiol 85, 1063-1069.

Suter PM, Schutz Y \& Jéquier E (1992) The effect of ethanol on fat storage in healthy subjects. New Engl J Med 326, 983-987.

Thun MJ, Peto R, Lopez AD, et al. (1997) Alcohol consumption and mortality among middle-aged and elderly U.S. adults. New Engl J Med 337, 1705-1714.

Tremblay A, Buemann B, Thériault G \& Bouchard C (1995a) Body fatness in active individuals reporting low lipid and alcohol intake. Eur J Clin Nutr 49, 824-831.

Tremblay A, Wouters E, Wenker M, et al. (1995b) Alcohol and a high-fat diet: a combination favoring overfeeding. Am J Clin Nutr 62, 639-644.

Weir JB (1949) New methods for calculating metabolic rate with special reference to protein metabolism. J Physiol 109, 1-9.

Westerterp KR \& Bouten CVC (1997) Physical activity assessment: comparison between movement registration and doubly labeled water method. Z Ernährungswiss 36, 263-267.

Westerterp KR \& Goris AHC (2002) Validity of the assessment of dietary intake: problems of misreporting. Curr Opin Clin Nutr Metab Care 5, 489-493.

Westerterp KR \& Meijer EP (2001) Changes in physical activity patterns with age: a physiological perspective. J Gerontol 56A, $7-12$.

Westerterp-Plantenga MS \& Verwegen CRT (1999) The appetizing effect of an alcohol aperitif in overweight normal weight humans. Am J Clin Nutr 69, 205-212. 